American Journal of Pharmaceutical Education 2021; 85 (6) Article 8415.

\title{
REVIEW
}

\section{A Scoping Review of Antimicrobial Stewardship Teaching in Pharmacy Education Curricula}

\author{
Ziad G. Nasr, PharmD, ${ }^{\mathrm{a}}$ Duha M. Abbara, PharmD, ${ }^{\mathrm{a}}$ Kyle J. Wilby, PharmD, PhD ${ }^{\mathrm{b}}$ \\ ${ }^{a}$ Qatar University, College of Pharmacy, Doha, Qatar \\ ${ }^{\mathrm{b}}$ University of Otago, School of Pharmacy, Aotearoa, New Zealand \\ Submitted October 5, 2020; accepted March 7, 2021; published June 2021.
}

Objective. To conduct a scoping review of the existing literature to identify published studies on innovative teaching and assessment practices for antimicrobial stewardship in the Doctor of Pharmacy curriculum and to provide a foundation for future scholarly research in this important area.

Findings. Seven studies were found that met the inclusion criteria. Two of the studies explored the extent, content, and methods of delivery of antimicrobial stewardship, four studies described elective courses in antimicrobial stewardship, and one study described an interprofessional module. Most studies were conducted in the United States. Several pharmacy schools in the UK and the US incorporated antimicrobial stewardship teaching into their curriculum. Learning objectives for the elective courses focused on guidelines issued by the Infectious Diseases Society of America (IDSA) and the Society for Healthcare Epidemiology of America (SHEA), antimicrobial stewardship literature appraisal and principal application. The most used teaching strategy was didactic lectures, followed by case studies. Active-learning approaches like simulators, problem-based learning, and presentations were also used but to a lesser extent.

Summary. Antimicrobial stewardship curricular reform may be influenced by the timing of the course, teaching approaches, and performance assessment of students. Antimicrobial stewardship learning should be a required of all pharmacy students. The scarcity of scholarly activity in the teaching of and assessment of learning in antimicrobial stewardship suggests that curricular planning should be guided by national or international organizations to ensure pharmacy students learn such important material.

Keywords: antimicrobial stewardship, pharmacy curriculum, pharmacy education

\section{INTRODUCTION}

Antimicrobial resistance leads to infections, resulting in increased morbidities, mortalities, and longer hospital stays, and is therefore a major global public health problem. ${ }^{1}$ The Centers for Disease Control and Prevention (CDC) in the United States proposed five core actions to take to combat antimicrobial resistance: infection prevention and control, tracking and data, vaccine and medication development, environment and sanitation, and antibiotic use and access. ${ }^{2}$ Antimicrobial stewardship is a coherent set of actions with the intent of promoting appropriate use of antimicrobials. ${ }^{3}$ Antimicrobial stewardship is implemented through antimicrobial stewardship programs, where a multidisciplinary team works together to achieve the ultimate goal of optimizing antibiotic use while ensuring proper patient care. ${ }^{3}$ Antimicrobial stewardship has been shown to increase cure rates in infectious diseases and decrease

Corresponding Author: Ziad G. Nasr, College of Pharmacy, Qatar University, PO Box 2713, Doha, Qatar. Tel: + 974-44035633. Email: znasr@qu.edu.qa treatment failures, adverse effects, antibiotic consumption, antibiotic resistance, and hospital costs. ${ }^{4-6}$

Antimicrobial stewardship teams are multidisciplinary in nature and should include infectious disease physician, an, a clinical pharmacist trained in infectious diseases, a clinical microbiologist, information technology personnel, an infection control professional, and a hospital epidemiologist. ${ }^{7}$ Pharmacist expertise is a core element of hospital antimicrobial stewardship programs as per CDC guidelines. ${ }^{8}$ In fact, the incorporation of an infectious disease-trained pharmacist within antimicrobial stewardship teams is associated with greater adherence to recommended antimicrobial stewardship practices, decreased inappropriate antimicrobial use, and decreased cost, as well as reduced mortality rates for sepsis and respiratory illnesses. ${ }^{9-11}$ The increased attention given to antimicrobial stewardship and the continuous efforts to counter antimicrobial resistance, along with the important role of pharmacists in driving desired outcomes, have resulted in increased demand for pharmacists trained in antimicrobial stewardship. ${ }^{12}$ 


\section{American Journal of Pharmaceutical Education 2021; 85 (6) Article 8415.}

Training of clinical pharmacists in the management of infectious diseases can be formal, such as through postgraduate residencies, certification programs, or practicing and gaining experience in an infectious disease patient care setting. ${ }^{13-15}$ As per the CDC guidelines, in institutions where there is no infectious disease clinical pharmacist, a general clinical pharmacist with infectious disease training can co-lead the antimicrobial stewardship program. ${ }^{8}$ In addition, pharmacists can gain exposure to various cases that require effective management of antimicrobial therapy in several settings, not just in infectious disease clinical settings, with varying responsibilities for and opportunities to practice antimicrobial stewardship. ${ }^{8,16-18}$ Therefore, new pharmacy graduates might be placed in positions where they need to fulfill certain duties related to antimicrobial stewardship even though they have not completed relevant postgraduate education in this area. It is in such positions where knowledge from a pharmacy curriculum that included antimicrobial stewardship is much needed. Curricular reform has also been suggested by practicing pharmacist stakeholders who see the need to better align antimicrobial stewardship teaching with the rapidly changing demands of real-life pharmacy practice. ${ }^{13}$ The American College of Clinical Pharmacy (ACCP) has a pharmacotherapy didactic curriculum toolkit that provides guidance on infectious disease, topics that need to be covered during pharmacy training. ${ }^{20}$ Also, the World Health Organization has a published curricula guide for health care education and training on antimicrobial resistance, including pharmacy education. ${ }^{21}$ A review conducted by Chahine and colleagues introduced a model to engage pharmacy students in antimicrobial stewardship which included the authors proposed timing for and means of introducing antimicrobial stewardship concepts to pharmacy students, residents, and fellows. ${ }^{22}$ Although the review gives insight into how to engage pharmacy students in antimicrobial stewardship, it does not provide information for practical curricular-based interventions. $^{22}$

Given the importance of antimicrobial stewardship and its increased role across multiple practice settings, pharmacy education should be reformed to strengthen students' understanding of antimicrobial stewardship concepts so that they can gain at least minimal competence prior to graduation to prepare them for any pharmacy practice work setting after graduation. The aim of this scoping review was to evaluate the existing literature on the inclusion of antimicrobial stewardship teaching and assessment within professional pharmacy curricula to provide a foundation for future scholarly research and to identify innovative teaching and assessment practices for antimicrobial stewardship programming.

\section{METHODS}

We followed the Joanna Briggs Institutes manual framework for scoping reviews when conducting this review. ${ }^{23}$ A protocol was developed a priori and followed without deviation. A scoping review methodology was chosen as this study aimed to explore the available evidence on antimicrobial stewardship education in pharmacy curriculum, identify and analyze knowledge gaps, clarify key concepts, and summarize the available evidence. The Population-Concept-Context for our scoping review included pharmacy curricula/students for the population, and antimicrobial stewardship/teaching methods as concepts in a global context.

To identify relevant studies, PubMed, EMBASE, CINAHL, and ERIC databases were searched. Pharmacy education journals, including the American Journal of Pharmaceutical Education, Currents in Pharmacy Teaching and Learning, and Pharmacy Education, were searched for pertinent studies. A manual search of the references cited in the included studies was also done to ensure inclusion of all relevant studies. Databases were searched from their inception date through February 2020. The search was restricted to English language publications. The search of specialized journals consisted only of the term antimicrobial stewardship in order to capture only relevant studies. Specific results from each database are included in Appendix 1.

Articles were included in the review if they discussed antimicrobial stewardship taught in pharmacy curricula in terms of content or delivery methods. Articles about postgraduate pharmacy education in antimicrobial stewardship, non-pharmacy students/curriculum, evaluation of students' perceptions, and knowledge of antimicrobial stewardship among students without considering curricular content or delivery were all excluded. Articles such as letters, editorials, commentaries, and conference abstracts were also excluded.

Search results were imported to Covidence (Veritas Health Innovation, Melbourne, Australia) online software. ${ }^{24}$ Titles and abstracts were screened for eligibility by one reviewer and approved by another reviewer. Two reviewers independently performed full text screening and met to review their findings. Disagreements were resolved through discussion. Data extraction was done by one reviewer using an extraction tool that included the following: study information (ie, title, author, setting and date), methodology, study design, key findings, implementation and prevalence of antimicrobial stewardship concepts, and teaching methodologies used.

A PRISMA flowchart (Figure 1) was used to describe the screening process of the studies. Findings were reported 


\section{American Journal of Pharmaceutical Education 2021; 85 (6) Article 8415.}

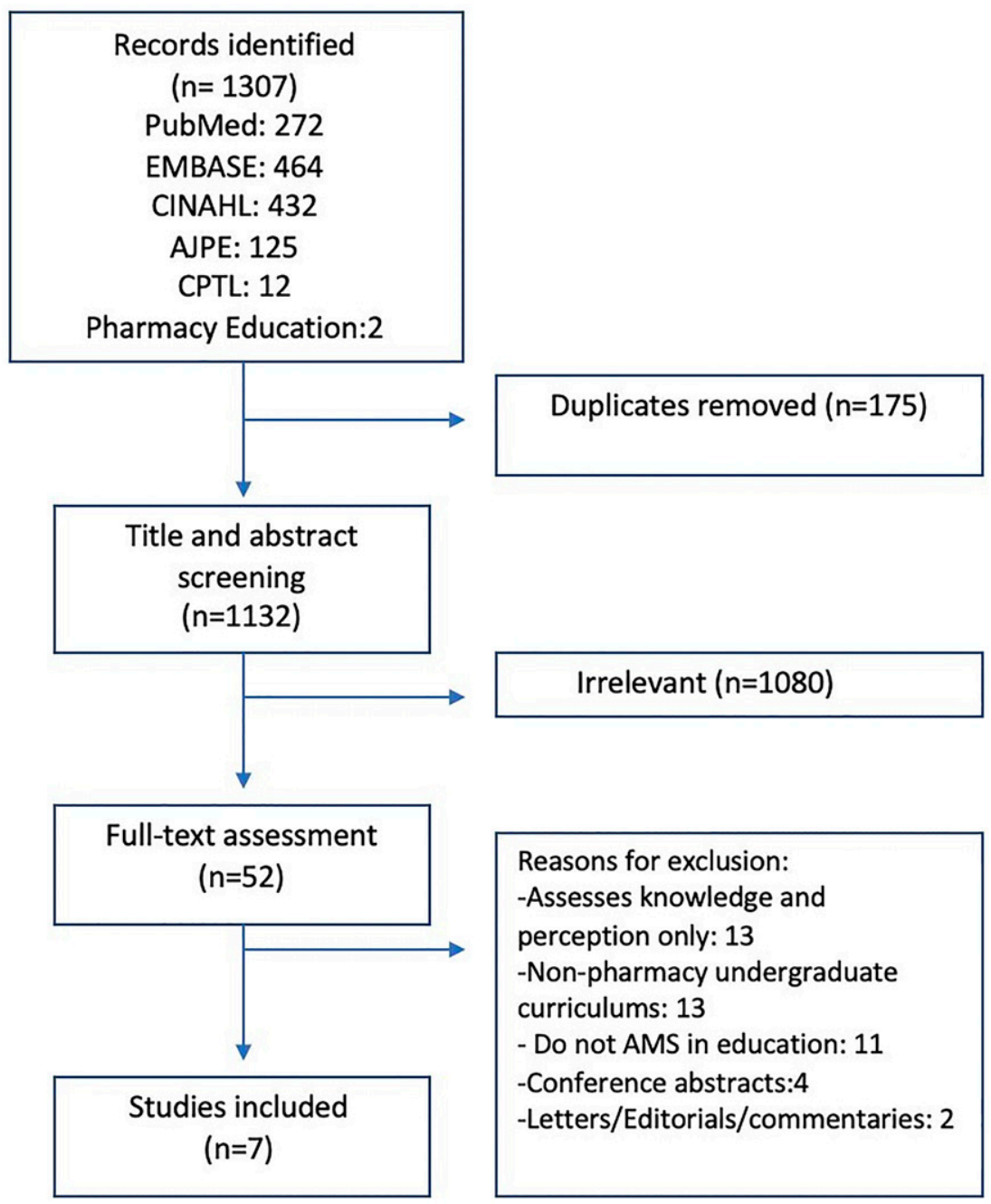

Figure 1. PRISMA Flowchart Illustrating the Process Used for Selecting Articles for a Review of Antimicrobial Stewardship Teaching in Pharmacy Education

in a tabular form. Descriptive analysis was used to describe both qualitative and quantitative data. For antimicrobial stewardship elective modules, learning objectives, methods for delivery, and assessments were reported. Institutional review board approval was not needed for this study.

\section{RESULTS}

A total of 1307 studies were identified from the search (Figure 1). After removing duplicates and screening titles and abstracts, 52 articles were eligible for full-text screening. After applying eligibility criteria to the full texts, seven articles were determined to be eligible for inclusion in the review. Two of the seven studies reported on the content of antimicrobial stewardship teaching in pharmacy schools in two different countries, the United States, and the United Kingdom. ${ }^{25,26}$ Data are presented in Table 1. Four studies described the design and implementation of an antimicrobial stewardship elective course, ${ }^{27-30}$ and one study described an interprofessional antimicrobial stewardship module embedded into the pharmacy curriculum. ${ }^{31}$ The learning objectives and methods of delivery and assessment for each study are reported in Table 2 . After synthesizing the data in the seven studies identified, we were able to separate findings into content and pedagogy.

\section{Content}

Two cross-sectional studies using online surveys were conducted to investigate the content and delivery of antimicrobial stewardship in the United Kingdom and the United States, respectively (Table 2) ${ }^{25,26}$ Antimicrobial stewardship education was incorporated in $86.6 \%(n=15)$ of UK pharmacy schools and $68.1 \%(\mathrm{n}=116)$ of US pharmacy programs. ${ }^{25,26}$ Some antimicrobial stewardship principles were more emphasized than others in the required curriculum, for instance, intravenous to oral conversion, legislative issues, rapid diagnostic testing, and source retrieval were 
American Journal of Pharmaceutical Education 2021; 85 (6) Article 8415.

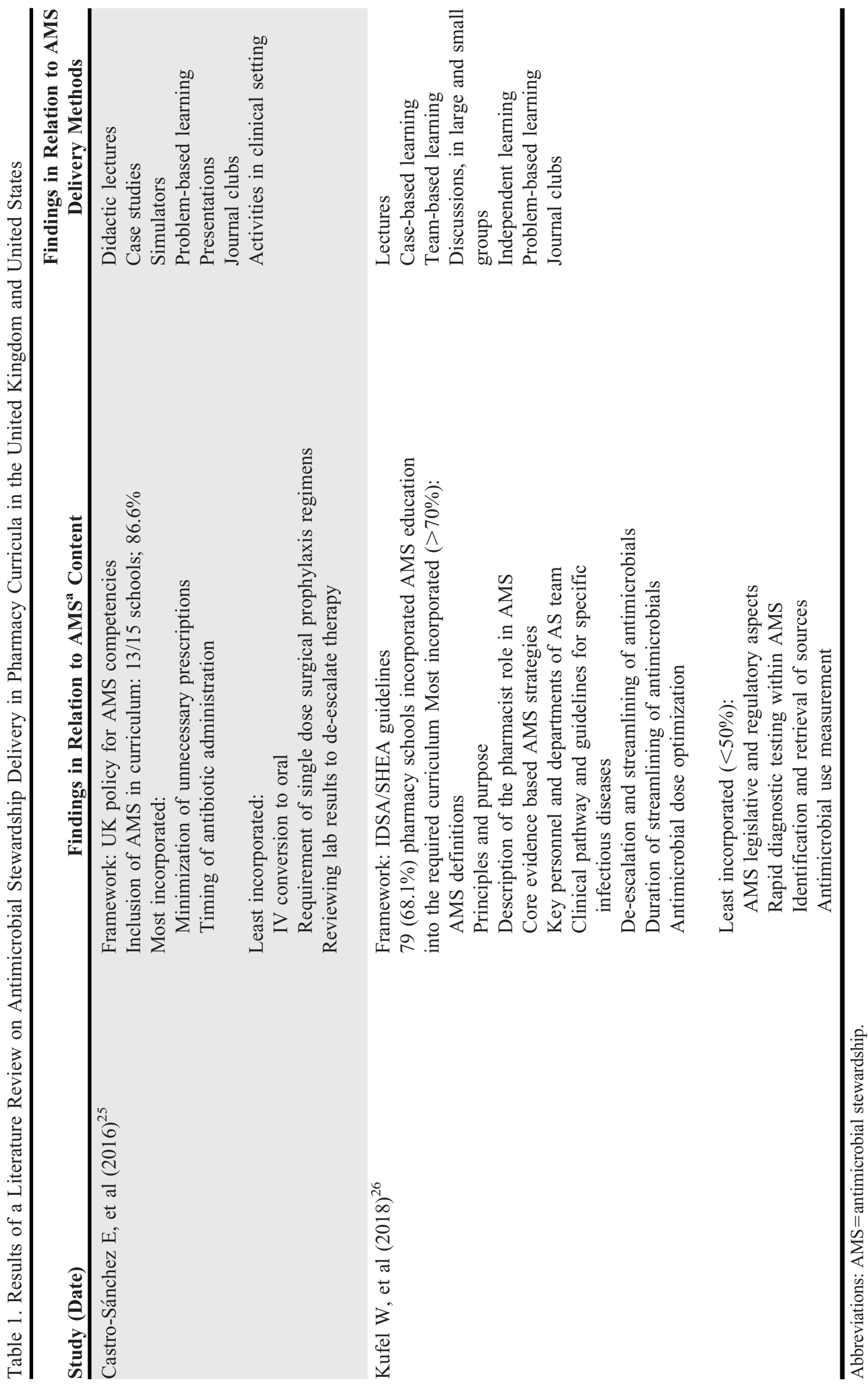


American Journal of Pharmaceutical Education 2021; 85 (6) Article 8415.

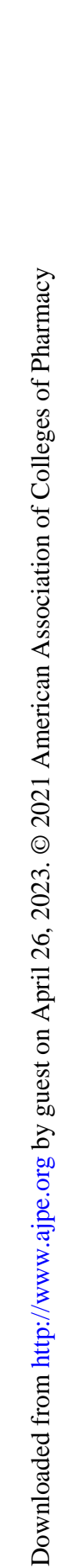
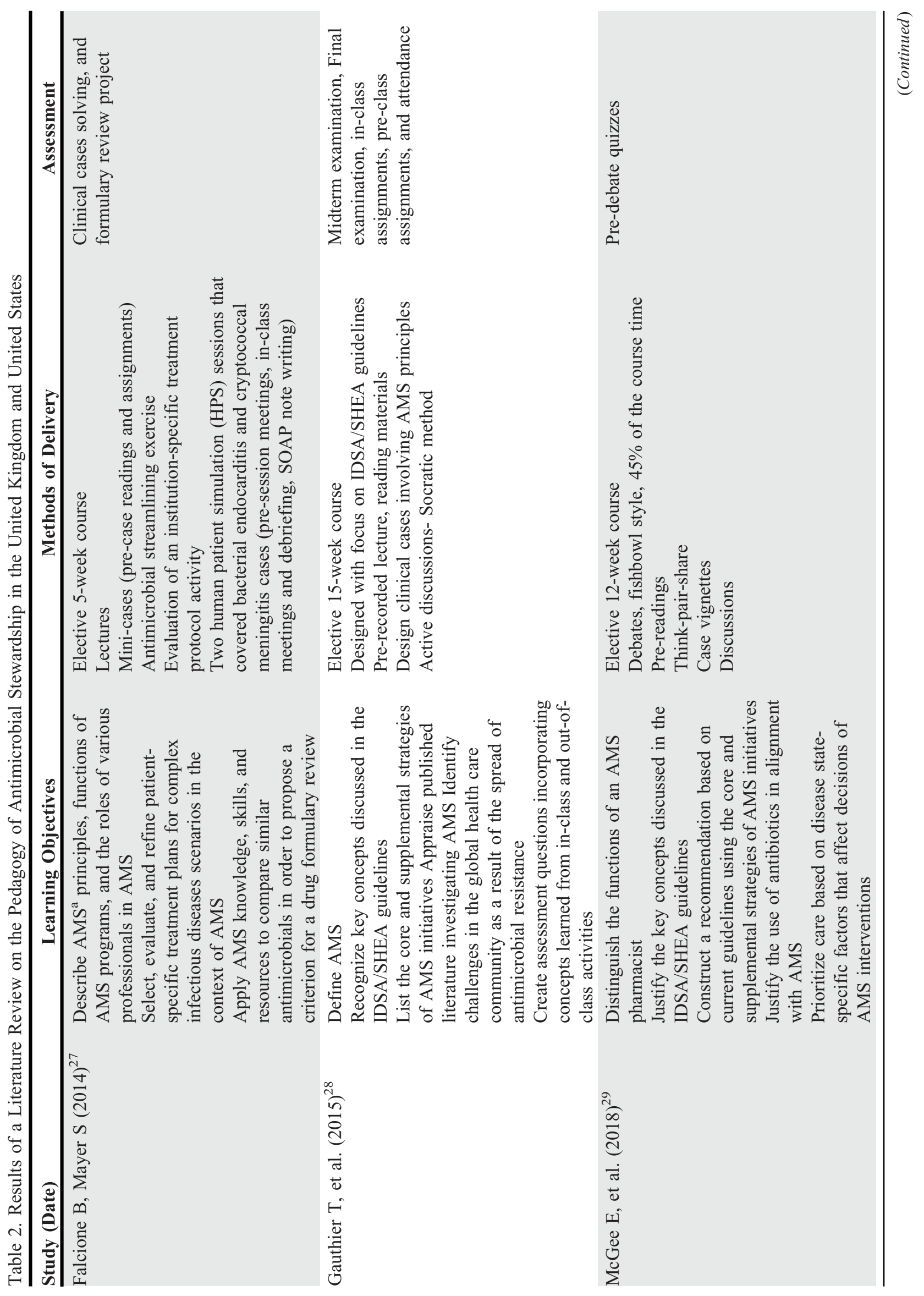

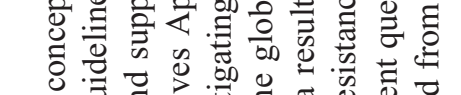

ص

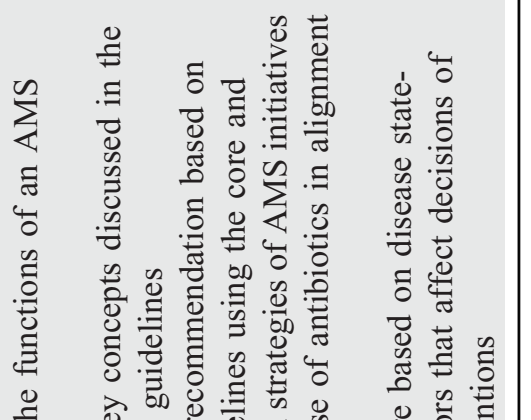

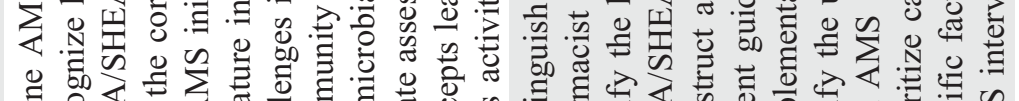

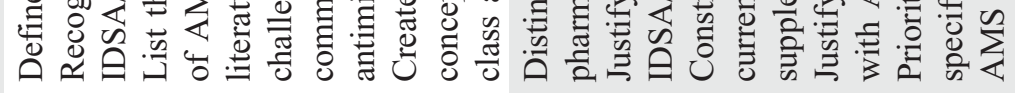

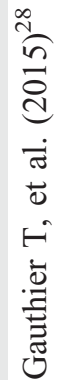

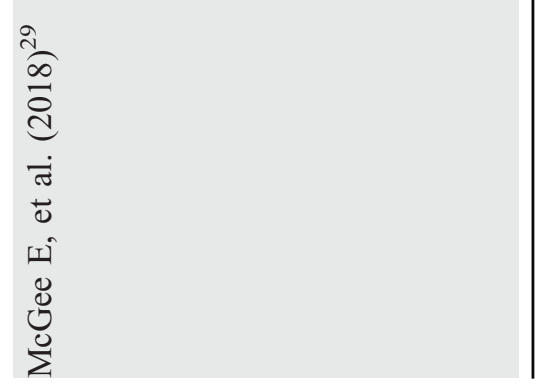


American Journal of Pharmaceutical Education 2021; 85 (6) Article 8415.

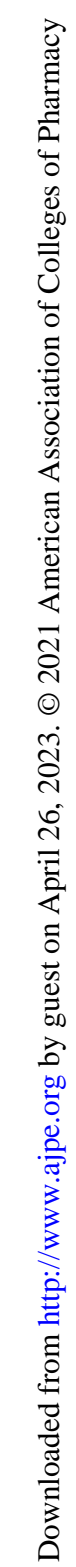
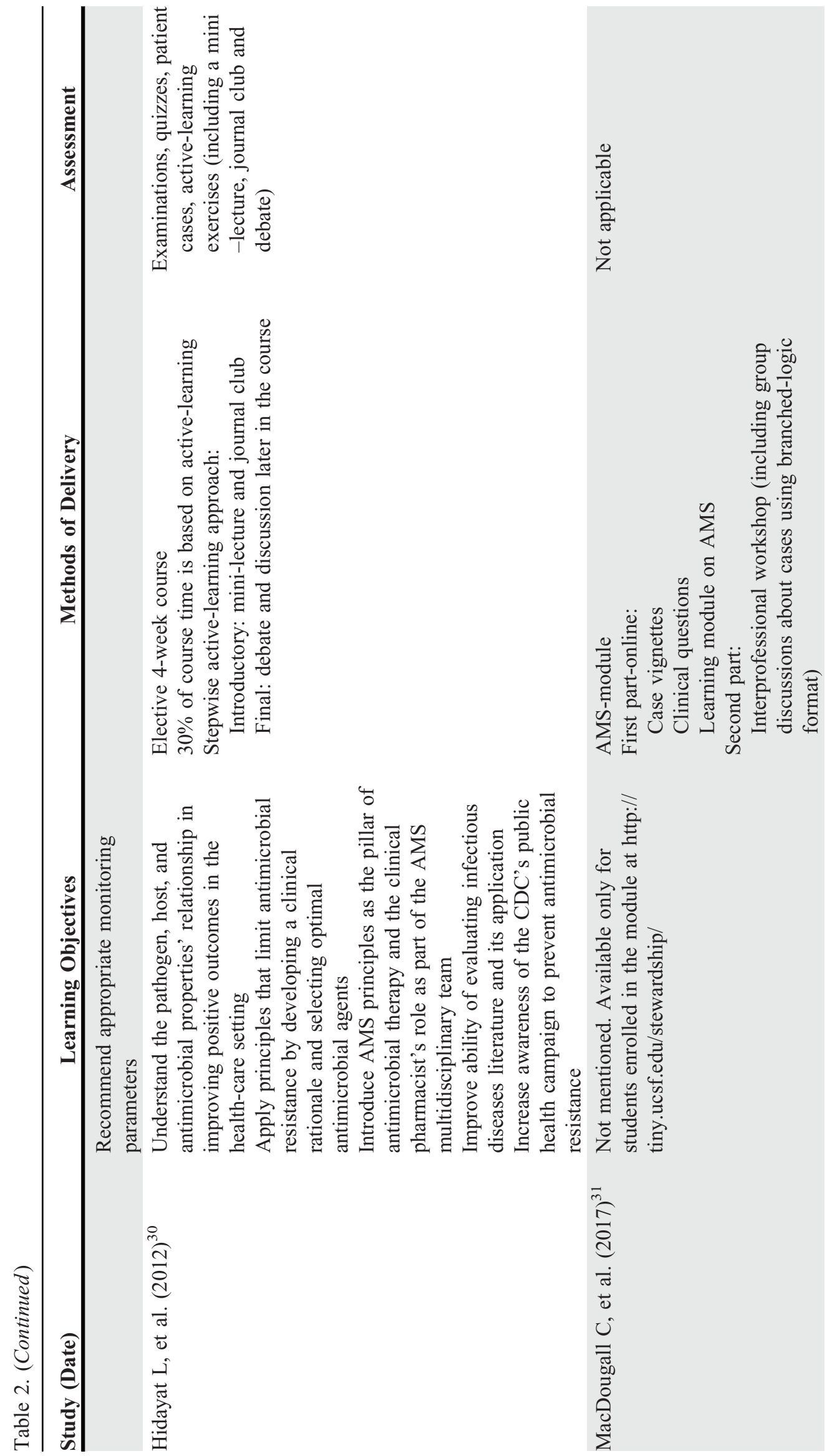


\section{American Journal of Pharmaceutical Education 2021; 85 (6) Article 8415.}

among the lesser discussed principles in pharmacy schools compared with the role of pharmacist in antimicrobial stewardship for instance. Also, the level and extent of exposure of students to antimicrobial stewardship topics differed by pharmacy school. ${ }^{25,26}$ The authors of both studies emphasized the importance of standardization in antimicrobial stewardship education.

The description of antimicrobial stewardship curricular content in the study by Castro-Sánchez and colleagues was based mainly on the UK policy for recommended principles for students to learn. ${ }^{32}$ The curricular content on antimicrobial stewardship in the study by Kufel and colleagues was based on the Infectious Diseases Society of America (IDSA) guidelines on teaching strategies, which are divided into core and supplemental topics. ${ }^{7}$

\section{Pedagogy}

The most frequently used teaching strategy for antimicrobial stewardship was didactic lectures, presented mostly in the form of case studies. The use of active-learning methods (eg, simulations, student presentations, journal clubs) was also used in both US and UK schools, but to a lesser extent than didactic teaching.

As for the elective antimicrobial stewardship courses, learning objectives, methods of delivery, and assessment methods are reported in Table 2. All electives were offered to third-year pharmacy students except for the one described in Gauthier and colleague's study which was open to both second- and third-year pharmacy students. Antimicrobial stewardship teaching approaches in these courses included active-learning strategies, self-learning, online learning, and interprofessional learning.

Active-learning strategies, such as clinical case studies and small and large group discussions, were present in all of the elective courses. Clinical cases were delivered using diverse approaches, such as human patient simulation, ${ }^{27}$ flipped classrooms, ${ }^{30}$ and classical case vignettes. ${ }^{28,30}$ Other active-learning strategies used were presentations, journal clubs, workshops, and debates.

Self-learning was also employed through assigned prereadings and prerecorded lectures. Courses that used these approaches devoted most of the in-class time to activelearning activities and workshops. ${ }^{28,29,31}$ In one of the studies, self-directed learning was largely done over an online platform. ${ }^{31}$ This activity involved interprofessional education through live interactions between pharmacy and medical students in a workshop one week post the online educational session. Interprofessional education concepts were also emphasized in the teaching intervention described by Falcione and colleague, where the multidisciplinary environment include simulated infectious disease physicians and nurses. ${ }^{26}$ Furthermore, the course incorporated a visit to a clinical microbiology laboratory in a hospital where students learned about the role of a clinical microbiologist. ${ }^{26}$

Learning objectives of the included antimicrobial stewardship elective courses ${ }^{27-30}$ are described in Table 2. Two of the courses included learning objectives related to IDSA antimicrobial stewardship guidelines. ${ }^{28,29}$ Three of the courses had objectives aimed at improving students' ability to critically evaluate and implement literature related to antimicrobial stewardship and infectious diseases. ${ }^{27,28,30}$ All of the courses included learning objectives on the application of antimicrobial stewardship principles (eg, selection and evaluation of antimicrobials, recommendations, and care plans). ${ }^{25-31}$

\section{DISCUSSION}

This study reviewed the available literature on antimicrobial stewardship content included in the pharmacy curriculum and teaching methods used to teach it. Seven articles were included. Of these, two studies looked into the curricular content in and methods of delivery for antimicrobial stewardship in pharmacy in the United Kingdom and United States, four articles described antimicrobial stewardship pharmacy electives, and one article described the implementation of an interprofessional antimicrobial stewardship module. ${ }^{25-31}$ All studies were conducted in the United States except for the one by Castro-Sánchez and colleagues, which was conducted in the United Kingdom. ${ }^{25-31}$ The results of the cross-sectional studies showed that the implementation of antimicrobial stewardship education in pharmacy curriculum is not consistent throughout US and UK pharmacy schools. This is consistent with IDSA's findings regarding the inconsistency and high variability in training of pharmacy students on optimal antimicrobial use for prescribers. ${ }^{33}$ This indicates that the problem of inconsistency extends beyond the scope of pharmacy education. This finding suggests that curricular planning for antimicrobial stewardship should be guided by national or international organizations in order to ensure the future pharmacy workforce is prepared to tackle antimicrobial resistance and other antimicrobial stewardship components in practice. An example of an effective strategy that could be followed is the World Health Organization's curriculum guidance on patient safety. ${ }^{34}$ International or national organizations could work on similar guidance for antimicrobial stewardship, yet specific attention should be paid to introducing concepts at different training levels (eg, undergraduate, postgraduate, and continuing professional development). 


\section{American Journal of Pharmaceutical Education 2021; 85 (6) Article 8415.}

Interestingly, antimicrobial stewardship was taught as an elective course in four of the articles identified. This finding poses the question of whether antimicrobial stewardship concepts should be a required curricular component or students should be allowed to choose whether they wish to study these concepts by completing an elective course). As antimicrobial resistance is continuing to grow worldwide and pharmacists have been shown to have a major role in rationalizing antimicrobial use, it could easily be argued that antimicrobial stewardship concepts should be taught as required curricular components to all future pharmacists. The extent of this content (ie, minimum material for inclusion), however, would benefit from further guidance by national and/or international organizations above and beyond what is currently provided by the ACCP and WHO. ${ }^{20,21}$ As stated previously, these guides focus on the possible inclusion of antimicrobial stewardship concepts in the pharmacy curriculum but guidance is nonspecific and could be better informed based on the results of this review. ${ }^{20,21}$

This review provides guidance regarding antimicrobial stewardship curricular reform. The first aspect to look at is timing. Based on the placement of the identified elective courses in the curriculum, antimicrobial stewardship was likely taught to students after they had gained foundational knowledge in infectious diseases and completed some introductory practice experiences. This strategy of placing content later in the program but just prior to APPEs rather than during the early years of study may be beneficial. A second important consideration in curricular planning is pedagogy. This review identified active learning as the preferred strategy for students to achieve the necessary learning outcomes. ${ }^{27,30}$ As learning commonly occurs by doing, designing activities for students to participate in (eg, simulations, case discussions, educational games) may be an effective strategy to promote mastery of antimicrobial stewardship skills. Specific learning strategies would need to be weighed against the available resources of the program and/or department. Finally, methods of assessment must be considered to ensure students are achieving the intended learning outcomes. This review primarily identified only knowledge assessment and therefore should be a priority of programs to explore the utility and effectiveness of other types of assessments (eg, case-based application, performancebased assessment, observation) in promoting learning and ensuring students are successfully gaining antimicrobial stewardship skills.

Despite providing an initial map of the scholarly literature on antimicrobial stewardship educational interventions for pharmacy students, the findings from this review should be interpreted in light of some limitations. The primary limitation of this review is the dependency on published literature to answer the research questions. In other words, some pharmacy programs may be including antimicrobial stewardship teaching in their curriculum but are not reporting it in the literature. The pharmacy education literature typically focuses on innovation and novel ways to teach and assess learning, and this may be why studies were primarily limited to elective course descriptions. Second, the review did not account for training that would occur as partofexperiential learning during practice-based experiences. Students may gain antimicrobial stewardship competencies during these experiences, but the impact of this training is not known. Despite these limitations, this review identified the need for better guidance on antimicrobial stewardship training within the pharmacy curriculum and presented several initial considerations (eg, timing, pedagogy, assessment) for curricular reform.

\section{CONCLUSION}

This review identified various ways in which pharmacy programs teach and assess antimicrobial stewardship concepts in pharmacy students, but only identified sparse literature contributing to new and innovative teaching and assessment techniques for development of antimicrobial stewardship development and delivery of antimicrobial stewardship curricula. These findings support the notion that curricular guidance should be driven by national or international organizations, especially given the increasing threat of antimicrobial resistance and the essential preventative role of pharmacists. Academics working in this area should be encouraged to share their experiences, test their educational interventions, and evaluate the effectiveness of teaching in this area. Doing so will allow for evidencebased implementation of antimicrobial stewardship curricula that should help to better train the pharmacy workforce and ultimately lead to improved patient care.

\section{REFERENCES}

1. World Health Organization. Antimicrobial resistance. https://www. who.int/news-room/fact-sheets/detail/antimicrobial-resistance. 2021. Accessed May 20, 2021.

2. Centers for Disease Control and Prevention. Antibiotic resistant threats in the United States. https://www.cdc.gov/drugresistance/pdf/ threats-report/2019-ar-threats-report-508.pdf. 2019. Accessed May 20, 2021.

3. Dyar O, Huttner B, Schouten J, Pulcini C. What is antimicrobial stewardship? Clin Microbiol Infect. 2017;23(11):793-798.

4. Davey P, Marwick C, Scott C, et al. Interventions to improve antibiotic prescribing practices for hospital inpatients. Cochrane Database Syst Rev. 2017;2(2):CD003543. doi: 10.1002/14651858.

5. Karanika S, Paudel S, Grigoras C, Kalbasi A, Mylonakis E. Systematic review and meta-analysis of clinical and economic outcomes from the implementation of hospital-Based Antimicrobial Stewardship programs. Antimicrob Agents Chemother. 2016;60(8):4840-4852. 


\section{American Journal of Pharmaceutical Education 2021; 85 (6) Article 8415.}

6. Ziglam H, Al-Madhoun E, Ahmedullah H, et al. An antimicrobial stewardship programme in a general hospital in Qatar: first year experience. In: ECCMID; April 21-24 2018; Madrid, Spain.

7. Dellit T, Owens R, McGowan J, et al. Infectious Diseases Society of America and the Society for Healthcare Epidemiology of America guidelines for developing an institutional program to enhance Antimicrobial Stewardship. Clin Infect Dis. 2007;44(2):159-177.

8. Centers for Disease Control and Prevention. The Core Elements of Hospital Antibiotic Stewardship Programs. https://www.cdc.gov/ antibiotic-use/healthcare/pdfs/hospitalcore-elements-H.pdf. 2019. Accessed May 20, 2021.

9. Bessesen M, Ma A, Clegg D, et al. Antimicrobial Stewardship Programs: comparison of a program with infectious diseases pharmacist support to a program with a geographic pharmacist staffing model. Hosp Pharm. 2015;50(6):477-483.

10. Yu K, Rho J, Morcos M, et al. Evaluation of dedicated infectious diseases pharmacists on antimicrobial stewardship teams. Am J Health Syst Pharm. 2014;71(12):1019-1028.

11. Al-Somai N, Al-Muhur M, Quteimat O, Hamzah N. The impact of clinical pharmacist and ID intervention in rationalization of antimicrobial use. Saudi Pharm J. 2014;22(6):516-521.

12. Justo J, Gauthier T, Scheetz M, et al. Knowledge and attitudes of doctor of pharmacy students regarding the appropriate use of antimicrobials. Clin Infect Dis. 2014; 59(suppl_3):S162-S169.

13. American Society for Health System Pharmacists. PGY2 Residencies. https://www.ashp.org/Professional-Development/ Residency-Information/Student-Residency-Guide/. 2021. Accessed May 20, 2021.

14. Board of Pharmacy Specialties. Infectious Diseases Pharmacy. https://www.bpsweb.org/bps-specialties/infectious-diseasespharmacy/. 2021. Accessed May 20, 2021.

15. Society of Infectious Diseases Pharmacists. Antimicrobial Stewardship Certificate Programs. https://sidp.org/Stewardship-

Certificate/. 2021. Accessed May 20, 2021.

16. American Society of Health System Pharmacists. ASHP statement on the pharmacist's role in antimicrobial stewardship and infection prevention and control. Am J Health Syst Pharm. 2010;67(7):575-577. 17. Garau, J, Bassetti, M. Role of pharmacists in antimicrobial stewardship programmes. IJCP. 2018;40(5):948-952.

18. Goff, DA. Antibiotic Stewardship: the health of the world depends on it. Hosp Pharm. 2018;53(4): 214-216.

19. Nasr Z, Higazy A, Wilbur K. Exploring the gaps between education and pharmacy practice on antimicrobial stewardship: a qualitative study among pharmacists in Qatar. Adv Med Educ Pract. 2018;10:287295.
20. Flannery AH, Soric MM, Benavides S, et al. 2019 Update to the American College of Clinical Pharmacy pharmacotherapy didactic curriculum toolkit. J Am Coll Clin Pharm. 2019;3(2):455-464. 21. Health workers' education and training on antimicrobial resistance: curricula guide. JAC Antimicrob Resist. 2019;1(3). doi: 10.1093/ jacamr/dlz073.

22. Chahine E, El-Lababidi R, Sourial M. Engaging pharmacy students, residents, and fellows in antimicrobial stewardship. J Pharm Pract. 2015;28(6):585-591.

23. Peters MDJ, Godfrey C, McInerney P, Munn Z, Tricco AC, Khalil H. Chapter 11: Scoping Reviews. In: Aromataris E, Munn Z (eds). JBI Manual for Evidence Synthesis, JBI, 2020. https://synthesismanual.jbi. global. https://doi.org/10.46658/JBIMES-20-12. Accessed May 20, 2021.

24. Covidence systematic review software. Melbourne, Australia: Veritas Health Innovation.

25. Castro-Sánchez E, Drumright L, Gharbi M, Farrell S, Holmes A. Mapping antimicrobial stewardship in undergraduate medical, dental, pharmacy, nursing and veterinary education in the United Kingdom. PLOS ONE. 2016;11(2):e0150056.

26. Kufel W, Jeffres M, MacDougall C, Cho J, Marx A, Williams D. Antimicrobial stewardship education in US colleges and schools of pharmacy. J Antimicrob Chemother. 2018; 73(8):2252-2258.

27. Falcione B, Meyer S. Development of an antimicrobial stewardship-based infectious diseases elective that incorporates human patient simulation technology. Am J Pharm Educ. 2014;78(8):151.

28. Gauthier T, Sherman E, Unger N. An elective course on antimicrobial stewardship. Am J Pharm Educ. 2015;79(10):157.

29. McGee E, Pius M, Mukherjee K. Assessment of structured classroom debate to teach an antimicrobial stewardship elective course. Curr Pharm Teach Learn. 2020;12 (2):220-227.

30. Hidayat L, Patel S, Veltri K. Active-learning implementation in an advanced elective course on infectious diseases. Am J Pharm Educ. 2012;76(5):87.

31. MacDougall C, Schwartz B, Kim L, Nanamori M, Shekarchian S, Chin-Hong P. An interprofessional curriculum on antimicrobial stewardship improves knowledge and attitudes toward appropriate antimicrobial use and Collaboration. OFID. 2017; ofw225.

32. Ashiru-Oredope D, Cookson B, Fry C, et al. Developing the first national antimicrobial prescribing and stewardship competences. $J$ Antimicrob Chemother. 2014;69(11):2886-2888.

33. Combating antimicrobial resistance: Policy recommendations to save lives. Clin Infect Dis. 2011;52(suppl 5):S397-S428.

34. World Health Organization. Multi-professional safety curriculum guide. https://www.who.int/patientsafety/education/curriculum/ Curriculum_Tools/en/.2011. Accessed May 20, 2021. 
American Journal of Pharmaceutical Education 2021; 85 (6) Article 8415.

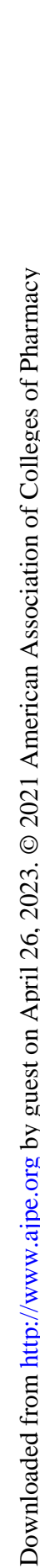

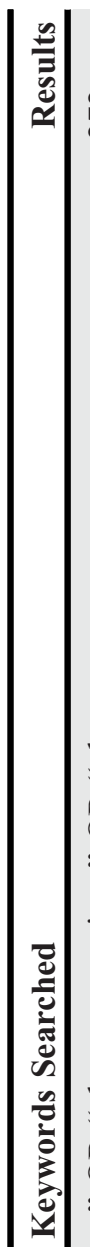

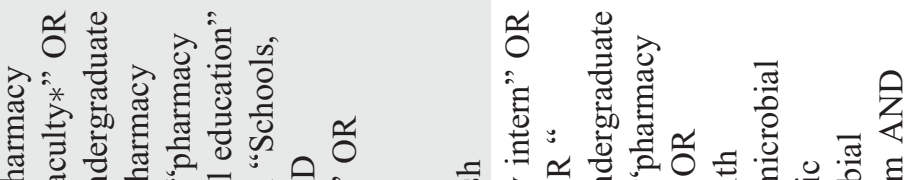

id

$\stackrel{\text { พ }}{+}$

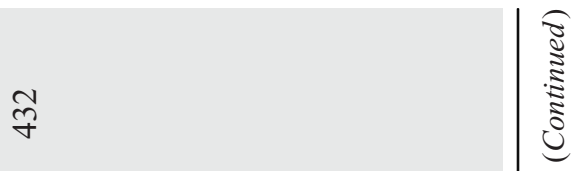

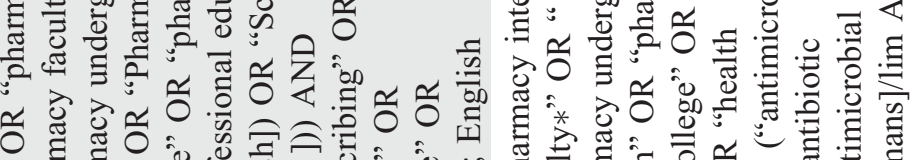

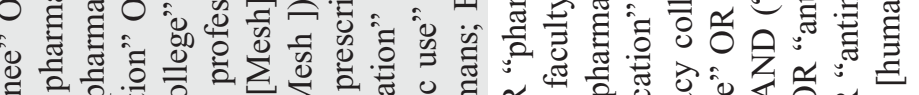

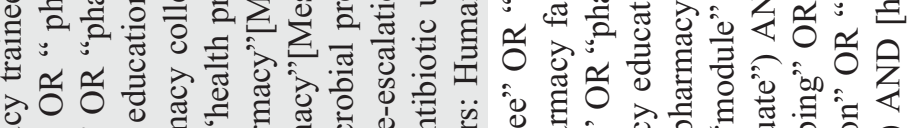
o

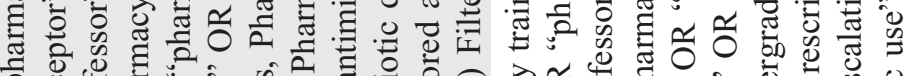

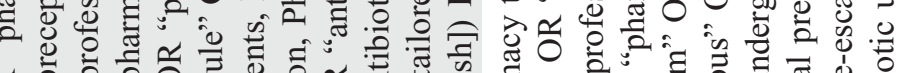

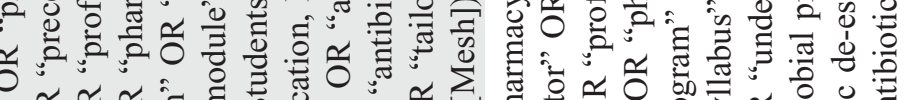

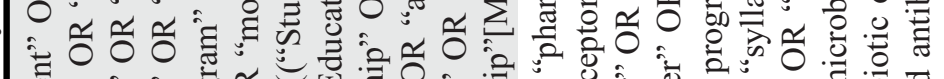
造

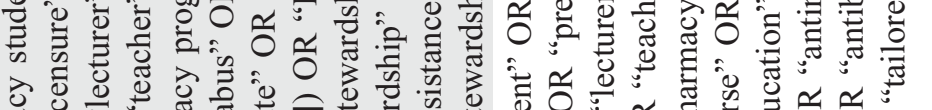

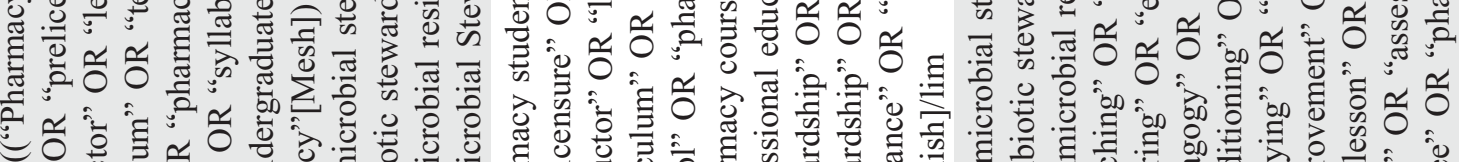

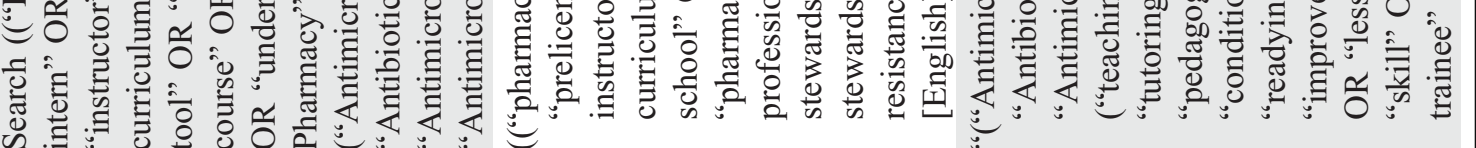

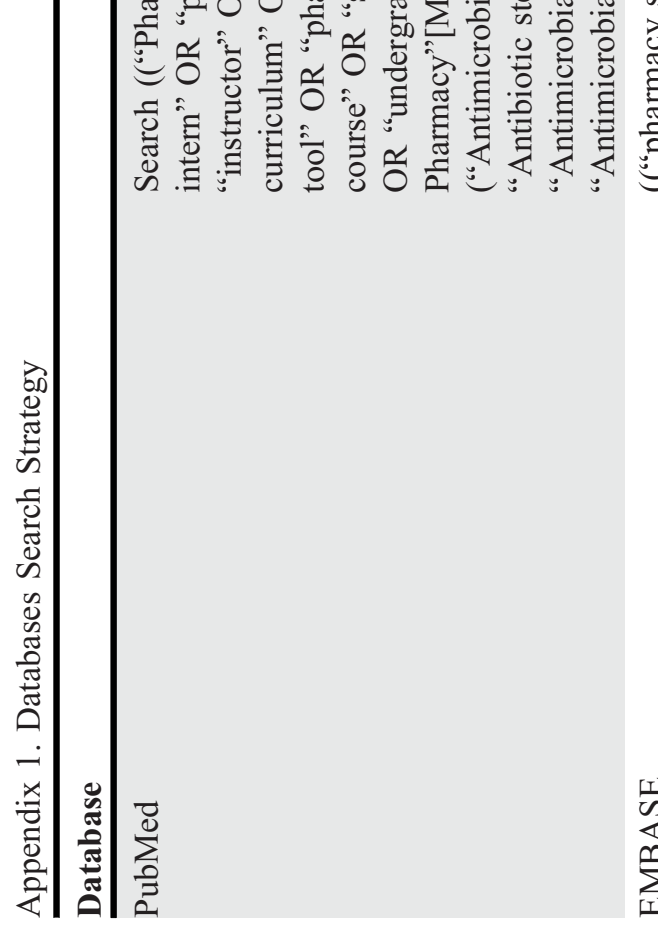

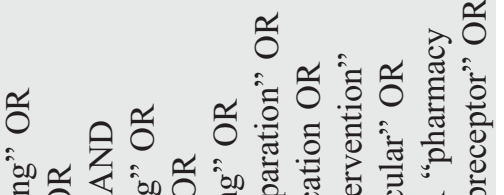

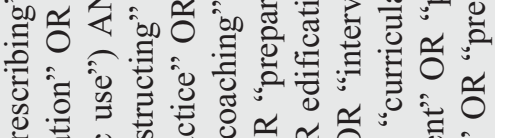

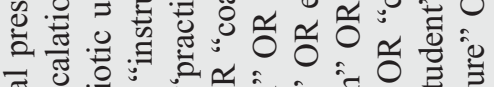
중

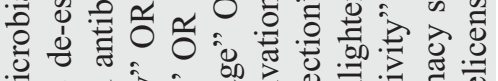

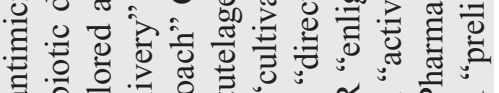

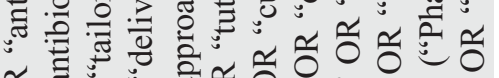
증 ज्ञ

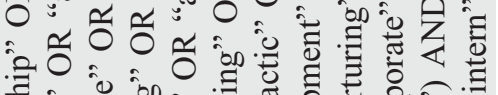

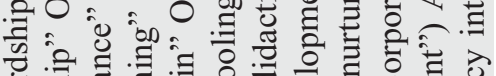

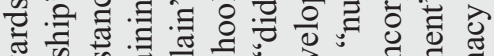

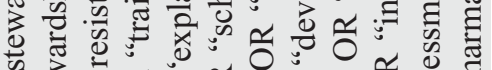

$\sum_{\substack{1 \\ \infty}}^{\infty}$

罗 
American Journal of Pharmaceutical Education 2021; 85 (6) Article 8415.

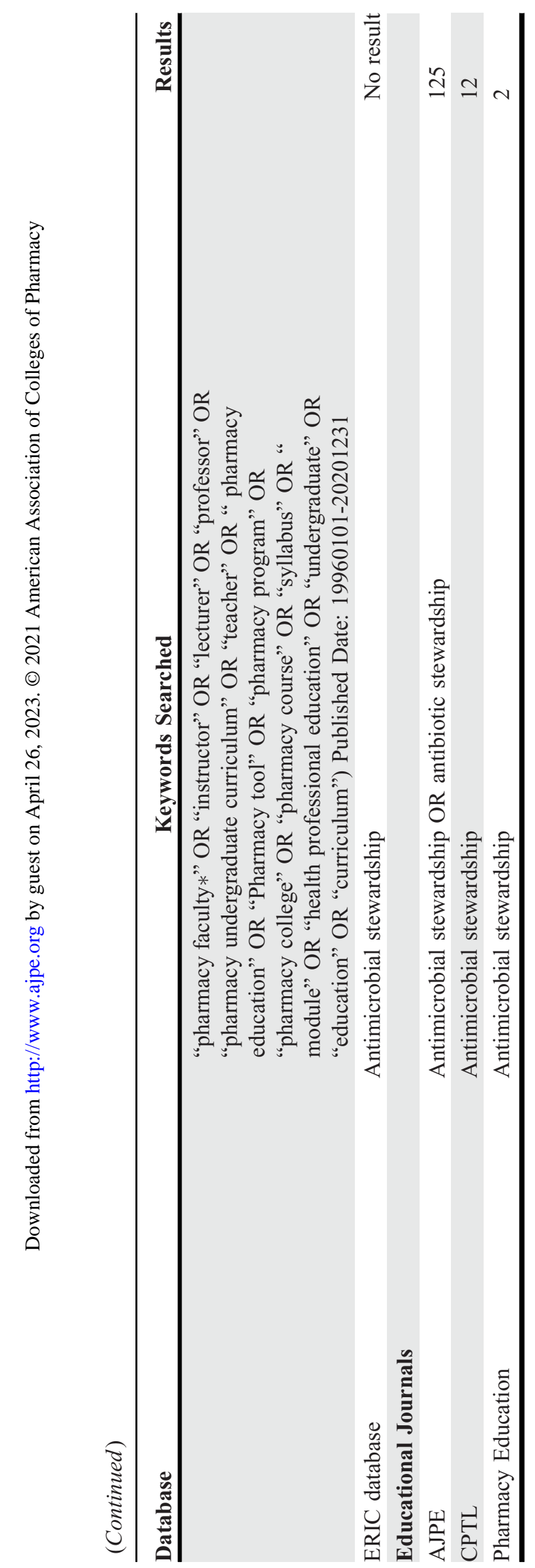

